\title{
Shared T Cell Recognition Sites on Human Histocompatibility Leukocyte Antigen Class II Molecules of Patients with Seropositive Rheumatoid Arthritis
}

\author{
Jorg Goronzy, Cornelia M. Weyand, and C. Garrison Fathman \\ Division of Immunology, Department of Medicine, Stanford University Medical Center, Stanford, California 94305
}

\begin{abstract}
Seropositive rheumatoid arthritis (RA) in adult and juvenile patients is associated with the serologic marker HLA-DR4. This association is incomplete; about one-third of the patients lack the disease-associated HLA-DR4 haplotype. The main biological function of class II molecules is to restrict the recognition of antigen by $T$ lymphocytes. We therefore tested the hypothesis that patients with seropositive $R A$ share $T$ cell recognition sites for an unknown antigen and that such $T$ cell "epitopes" are not identified by conventional serologic typing. We generated alloreactive human $T$ cell clones by stimulating peripheral blood lymphocytes of normal donors against a lymphoblastoid cell line from a juvenile patient with seropositive RA. A panel of clones that recognized only HLA-Dw14 cells on a panel of homozygous typing cells was used to analyze class II molecules of adult patients with seropositive RA. By inhibition studies using monoclonal antibodies, the epitopes recognized by the different clones could be further characterized and assigned either to DR- or to DQ-encoded cell surface products. By using four different clones, it was possible to identify Dw14-associated $T$ cell epitopes on all seropositive rheumatoid patients tested who typed HLA-DR4positive and also on all eight DR4-negative patients tested. Approximately one-half of nonrheumatoid DR4-positive donors carried one or more determinants recognized by these clones; the expression of these allodeterminants in DR4-negative nonrheumatoid patients was rare $(<\mathbf{1 0} \%)$. Thus, alloreactive human $T$ cell clones are powerful tools to define $T$ cell recognition sites on class II molecules that are not identified by conventional typing. Using $T$ cell clones with specificities for determinants expressed on Dw14 homozygous typing lines, we were able to demonstrate shared epitopes on cells of all patients tested with seropositive RA irrespective of their HLA-D or HLA-DR type. These data suggest that major histocompatibility complex class II antigens of RA patients might be much more homogeneous than demonstrated by the incomplete HLA-DR4 association.
\end{abstract}

Received for publication 24 June 1985 and in revised form 26 November 1985.

J. Clin. Invest.

(c) The American Society for Clinical Investigation, Inc.

0021-9738/86/03/1042/08 \$1.00

Volume 77, March 1986, 1042-1049

\section{Introduction}

The highly polymorphic cell surface glycoproteins encoded within the human major histocompatibility complex (MHC) ${ }^{1}$ serve to restrict the recognition of antigen by $\mathrm{T}$ cells $(1,2)$. Recognition of epitopes on nominal antigens, together with $\mathrm{MHC}$ determinants, is a necessary event for the activation of $\mathrm{T}$ lymphocytes. Several human diseases, most of which are characterized by autoimmune phenomena, have been associated with HLA determinants, in particular with MHC class II (HLA-D) molecules (3-5). These MHC class II molecules function as restriction elements for $\mathrm{T}$ helper cells (6). $\mathrm{T}$ helper cells are responsible for the initiation of immune responses against most antigens. Thus, the ability of the immune system to recognize and eliminate antigens is determined in part by the structure and function of the MHC molecules. "Inappropriate recognition" of antigen in association with the $\mathrm{T}$ cell recognition sites on MHC class II molecules could cause "abnormal" immune responses and might be the explanation for the association of certain autoimmune diseases with particular MHC Class II molecules.

Human MHC class II molecules have been defined and characterized by conventional serologic techniques as HLA-DR specificities, and by mixed lymphocyte reactions as HLA-D specificities. By cellular typing, the serologically defined HLADR4 specificity has been divided into five subgroups: Dw4, Dw10, Dw13, Dw14, and Dw15 (7-9). 60-80\% of patients with seropositive rheumatoid arthritis (RA) are HLA-DR4-positive (10-13). HLA-DR4 is the haplotype associated with the seropositive form of rheumatoid arthritis (RA) in many different ethnic groups (14-16) providing strong evidence that this haplotype may play a major role in susceptibility to the disease. An etiologic agent for RA has not been identified; thus the antigens that might be involved in triggering the disease are unknown. We hypothesized that the striking association of seropositive RA with HLA-DR4 is in part due to a specific polymorphism(s) within MHC class II molecules that can lead to an arthritogenic recognition of a yet unknown antigen by $T$ lymphocytes. It has been shown that functionally relevant alterations of $\mathrm{T}$ cell re-

1. Abbreviations used in this paper: EBV, Epstein-Barr virus; HTC, homozygous typing cells; IL-2, interleukin 2; MHC, major histocompatibility complex; PLT, primed lymphocyte typing; RA, rheumatoid arthritis. 
striction elements are reflected by changes in $T$ cell epitopes recognized as allodeterminants (17). Because there is no identified disease-causing antigen for RA, we generated human alloreactive $\mathrm{T}$ cell clones which were then used to analyze class II molecules of cells from patients with RA. We developed a panel of $T$ cell clones that defined distinct HLA-DR4-related $T$ cell epitopes on HLA-DR- and HLA-DQ-encoded molecules. On homozygous typing cells, a panel of these clones recognized determinants present only on Dw14-positive cell lines. When these clones were tested on patients with seropositive RA, one or more of these Dw14-related determinants could be identified on all patients tested ( $n=23$ ) irrespective of their HLA-D and HLADR type. These data suggest that patients with RA share $T$ cell epitopes that are not recognized by conventional typing.

\section{Methods}

Preparation of peripheral blood lymphocytes (PBL). Heparinized venous blood was diluted with an equal volume of RPMI 1640 medium and separated by density gradient centrifugation on Ficoll-Hypaque. Cells within the interface layer were washed three times and resuspended in RPMI 1640 supplemented with $10 \%$ fetal calf serum.

Generation of alloreactive $T$ cell clones. Alloreactive $T$ cell clones were isolated and maintained as described (18). Briefly, $5 \times 10^{5} / \mathrm{ml}$ responder cells were stimulated by $5 \times 10^{5} / \mathrm{ml}$ irradiated $(10,000 \mathrm{rads})$ lymphoblastoid cells from a DR4 homozygous Dw4/Dw14 heterozygous patient with juvenile seropositive RA in $20 \mathrm{ml}$ of complete culture medium (RPMI 1640 supplemented with $10 \%$ fetal calf serum and $2 \mathrm{mM}$ L-glutamine). PBL of five different healthy donors were used as responders: four of them shared one DR4 haplotype with the stimulator, the fifth typed DR2, negative. Every 2 wk, bulk cultures were restimulated with $5 \times 10^{5} / \mathrm{ml}$ irradiated Epstein-Barr virus (EBV) blasts and tested for antigen responsiveness to the primary stimulators in a proliferation assay at day 4. 6-8 wk after initiation of the bulk culture, $T$ cells were cloned by the technique of limiting dilution. Viable $\mathrm{T}$ cells were plated ( 0.3 cell/well) in the presence of irradiated stimulators cells and $2 \mathrm{U} /$ well recombinant interleukin 2 (IL-2) (Cetus Corporation, Emeryville, CA) in 96-well flat-bottomed trays. Growing clones, observed microscopically, were transferred to 24-well Costar plates together with fresh antigen and IL-2-containing medium. Established T cell clones were maintained in IL-2-enriched complete medium and were restimulated with alloantigen at 2 -wk intervals.

$T$ cell proliferation assay. Proliferative responses of the alloreactive $T$ cell clones were determined in a thymidine incorporation assay as described (18). Briefly, rested T cells (10,000-20,000/well) were cocultured with irradiated EBV blasts $(30,000 /$ well) or irradiated PBL $(100,000 /$ well) in triplicate cultures in $200 \mu \mathrm{l}$ of complete culture medium in 96well round-bottomed trays (Linbro, Flow Laboratories, McLean, VA). Generally, the total culture time was $60 \mathrm{~h}$, for the last $12 \mathrm{~h}$, the cultures were pulsed with $1 \mu \mathrm{Ci}\left[{ }^{3} \mathrm{H}\right]$ thymidine/well. After harvesting the cultures on glass fiber filters, thymidine incorporation was measured by liquid scintillation spectroscopy. Results are expressed as the antigen-specific thymidine uptake (difference in counts per minute, $\Delta \mathrm{cpm}$ ), which was calculated by subtracting the mean counts per minute without responders from the mean counts per minute from responder-stimulator cocultures. Without added antigen, the cloned cells incorporated below $300 \mathrm{cpm}$; the counts per minute of stimulators alone were in the range of 500 1,500. Standard errors of the triplicate cultures were $<20 \%$. To allow comparison of the results obtained in different assays, Bin40, LS40, and autologous controls were included in all experiments and proliferative responses to the individual stimulators were expressed as a percent fraction of the maximum thymidine uptake induced by Bin 40 (relative response). A relative response of $<20 \%$ was defined as negative.

Target inhibition by monoclonal antibodies. Before the initiation of the culture, $3 \times 10^{4}$ irradiated stimulator cells were incubated with serial dilutions of monoclonal antibodies ( $5 \mu \mathrm{g} /$ well to $0.03 \mu \mathrm{g} /$ well). The fol-
Table I. Clinical Parameters of 23 Rheumatoid Patients

$\begin{array}{lc}\text { Age }(y r) & 54.3+15.4^{*} \\ \text { Male/female }(n) & 5 / 18 \\ \text { Duration of disease }(y r) & 11.2+7.6 \\ \text { Log latex } & 8.6+2.1 \\ \text { American Rheumatism } & \\ \quad \text { Association- functional class } & 2.6+0.8 \\ \text { Treatment }(n) & \\ \text { Gold } & 17 / 23 \\ \text { Penicillamine } & 11 / 23 \\ \text { Prednisone } & 17 / 23 \\ \text { Methotrexate } & 12 / 23 \\ \text { TLI } & 6 / 23\end{array}$

*Mean \pm SD.

lowing antibodies were used: L243, L227, L203 (provided by Dr. Ronald Levy, Stanford University), and P4.1 (provided by Dr. J. Hansen, Fred Hutchinson Cancer Center, Seattle, WA) are directed against monomorphic DR determinants. IV D12, an antibody against the HLA-DQ specificity, MB3, was a gift of Dr. R. Giles (University of Texas Southwestern Medical Center, Dallas, TX). B7/21 has been characterized to be specific for HLA-DP and was obtained from Dr. J. Trowbridge (Salk Institute, La Jolla, CA). Proliferative responses were measured as described. Percent response was calculated by dividing the antigen-induced thymidine uptake in the presence of monoclonal antibody by the uptake in the absence of antibody $\times 100$.

Cell lines. Homozygous typing cells (HTC) were generously provided by Dr. John Hansen and Dr. Erik Mickelson (Fred Hutchinson Cancer Center), Dr. Susan Hsu (Johns Hopkins Medical School, Baltimore, MD), and Dr. Nancy Reinsmoen (University of Minnesota, Minneapolis, MN).

Primed lymphocyte typing (PLT) reagents used for HLA-Dw14 typing were generated by primary stimulation against $\operatorname{Bin} 40$ as previously described $(19,20)$. Optimal discriminating results between Dw14-positive and Dw14-negative DR4-positive cells were obtained by coculturing 1 $\times 10^{4}$ primed lymphocytes and $1 \times 10^{5}$ irradiated PBL or $3-4 \times 10^{4}$ EBV blasts for $60 \mathrm{~h}$. HLA-DR typing for the specificities DR 1 through DRw10 was performed on nylon wool purified peripheral blood B lymphocytes or B lymphoblastoid cell lines (21).

Subjects. 23 patients treated at the Stanford Immunology Clinic fulfilled the American Rheumatism Association criteria for classical RA and were chosen for the study. All of them had a disease duration of $>1$ yr and were rheumatoid factor-positive. The control group consisted of randomly collected unrelated healthy donors, whose cells were HLADR typed. Additionally, 10 healthy individuals who were known to be DR4-positive were included in the controls.

Table II. HLA-DR Types of 23

Patients with Adult Seropositive RA

\begin{tabular}{lr}
\hline Type & \% \\
\hline DR1 & 26 \\
DR2 & 13 \\
DR3 & 4 \\
DR4 & 65 \\
DR5 & 17 \\
DRw6 & 4 \\
DR7 & 17 \\
DRw8 & 13 \\
DRw9 & 4 \\
DRw10 & 13 \\
\hline
\end{tabular}


Table III. Characterization of Human T Cell Clones on a Panel of Homozygous Typing Lines Representing Five Subtypes of the DR4 Family

\begin{tabular}{|c|c|c|c|c|c|c|c|c|}
\hline & HLA-D & T4-31 & GAl-5 & BA-1-7 & CB1-15 & $C 2-6$ & C2-1 & $\mathrm{Cl} 1-19$ \\
\hline & & $c p m$ & $c p m$ & $c p m$ & $c p m$ & $c p m$ & $c p m$ & $c p m$ \\
\hline JAH & 4,4 & $0^{*}$ & 0 & 0 & 0 & 126 & 333 & 249 \\
\hline BSM & 4,4 & 520 & 353 & 75 & 574 & 596 & 7 & 0 \\
\hline BOD & 4,4 & 752 & 0 & 0 & 290 & 710 & 156 & 0 \\
\hline AL10 & 10,10 & 504 & 0 & 625 & 15,413 & 0 & 40 & 0 \\
\hline TS10 & 10,10 & 160 & 205 & 32 & 18,557 & 419 & 0 & 0 \\
\hline KT2 & 13,13 & 22 & 506 & 122 & 310 & 45 & 30 & 0 \\
\hline JHA & 13,13 & 563 & 86 & 54 & 404 & 0 & 0 & 0 \\
\hline BIN 40 & 14,14 & 16,887 & 24,436 & 36,762 & 42,021 & 4,393 & 570 & 19,827 \\
\hline LS40 & 14,14 & 21,107 & 25,227 & 33,620 & 53,447 & 17,857 & 5,615 & 12,981 \\
\hline IST & 14,14 & 12,077 & 11,255 & 15,424 & 17,370 & 247 & 5,266 & 619 \\
\hline HAS 15 & 15,15 & 1,490 & 0 & 142 & 21 & 309 & 0 & 316 \\
\hline
\end{tabular}

* $1 \times 10^{4}$ cloned $\mathrm{T}$ cells were stimulated with $3 \times 10^{4}$ irradiated stimulator cells. Cultures were pulsed after $48 \mathrm{~h}$ with $1 \mu \mathrm{Ci}\left[{ }^{3} \mathrm{H}\right]$ thymidine and harvested after additional $12 \mathrm{~h}$. Results are expressed as net counts per minute defined by the proliferative response of the clone in the presence of stimulator cells minus counts per minute incorporated by stimulator cells alone.

\section{Results}

$H L A-D R$ phenotypes in patients with seropositive $R A$. The MHC class II antigens of 23 patients with classical RA were analyzed by conventional HLA-DR typing and by using alloreactive human $\mathrm{T}$ cell clones (see below). These patients were characterized clinically by a severe and progressive form of the disease. Most of them had failed disease-remitting therapy and had been treated with cytotoxic agents or total lymphoid irradiation (Table I). $65 \%$ of the patients typed DR4 (Table II), which is in accord with the known HLA-DR4 association of seropositive RA (11), and is more than twice the frequency of DR4 in a normal population (22). The expected compensatory decrease of other haplotypes was not seen for DR1, DRw8, and DRw10. DRw8 and DRw10 are rare specificities in a caucasian population; the frequency of DR 1 is $\sim 20 \%$ (22).

Generation and characterization of alloreactive $T$ cell clones. Long-term $\mathrm{T}$ cell lines were established from five different healthy donors (T:DR2,_; BA:DR4,4; CB:DR2,4; C:DR4,4; GA: DR4,w6). PBL from each of these donors were stimulated by a lymphoblastoid cell line derived from a patient with juvenile seropositive RA, who typed DR4 homozygous and Dw4/Dw14 heterozygous. Seven clones that proliferated in response to the original stimulator and Dw14 but not Dw4 HTC were then characterized on a panel of homozygous typing lines (HTC) (Table IV). Six of seven clones showed selective reactivity to Dw14-expressing cells: T4-31, GA1-5, BA1-7 recognized all three Dw14-expressing HTC. C2-1, C1-19, and C2-6 responded to only two out of the three Dw14 typing lines (Table III). Clone CB1-15 reacted against an epitope which was shared by cell lines positive for HLA-Dw14 and HLA-Dw10. None of the other six clones was reactive against non-HLA-Dw 14 specificities of the DR4 family (Table III) nor did any of the clones including CB115 react against non-DR4 HTC. None of these alloreactive clones responded to their autologous controls (data not shown).

The MHC class II molecules recognized by the alloreactive clones were further characterized by target inhibition studies with monoclonal antibodies against monomorphic determinants of HLA-DR, DQ, and DP molecules. As described recently, clone
T4-31 recognized an HLA-DR-encoded molecule and was blocked by a panel of anti-DR antibodies (18). Using the same panel of monoclonal antibodies, a similar but different inhibition pattern was obtained for clone GA1-5 suggesting that these two clones recognized spatially distinct epitopes on the HLA-DR molecule. The same panel of anti-DR antibodies (L227, L243, L203, P4.1) blocked the proliferative response of clone BA1-7 (Table VA) and CB1-15 (see Table VA and Fig. 2) and allowed the assignment of their $T$ cell recognition sites (epitopes) to DR encoded molecules. The determinant seen by clone $\mathrm{C} 1-19$ has been previously shown to be located on a DQ region product (18). Monoclonal antibody blocking studies demonstrated that the $T$ cell epitopes seen by clone C2-1 and clone 2-6 were also on DQ molecules (Table VA). Thus, the alloreactive human T cell clones allowed the definition of four $\mathrm{T}$ cell recognition sites (epitopes) on DR molecules and three $T$ cell epitopes on DQ molecules. The determinants defined by the seven clones described here were arbitrarily named as follows: DR-T1, DR-T2, DR-T3, and DR-T4 for the T cell epitopes encoded by the DRregion and DQ-T1, DQ-T2, and DQ-T3 for the $T$ cell target structures expressed by DQ molecules (Table VB).

Table IV. HLA Haplotypes of DR4-positive Homozygous Typing Lines

\begin{tabular}{lcllll}
\hline & A & B & DR & DQw & DRw53 \\
\hline JAH & 2 & 62 & 4,4 & 3 & + \\
BSM & 2 & 15 & 4,4 & 3 & + \\
BOD & 2 & 15 & 4,4 & 3 & + \\
AL 10 & 26,33 & 38 & 4,4 & 3 & + \\
TS 10 & 26 & 38 & 4,4 & 3 & + \\
KT2 & 2 & 51 & 4,4 & 3 & + \\
JHA & 31 & 51 & 4,4 & 3 & + \\
BIN 40 & 2,31 & 14,60 & 4,4 & 3 & + \\
LS40 & 1,26 & 27,60 & 4,4 & 3 & + \\
IST & 2 & 35 & 4,4 & blank & + \\
Has 15 & 2 & 15 & 4,4 & blank & $(+)$ \\
& & & & & + \\
\hline
\end{tabular}


Table V. Definition of DR- or DQ-encoded T Cell Epitopes Recognized by Seven Different T Cell Clones

\begin{tabular}{|c|c|c|c|c|c|c|c|c|}
\hline \multirow[b]{2}{*}{ A. Clone } & \multicolumn{4}{|c|}{ MAb anti-DR molecules } & \multicolumn{2}{|c|}{ MAb anti-DQ molecules } & \multicolumn{2}{|c|}{ MAb anti-DP molecules } \\
\hline & L243 & $\mathrm{L} 203$ & L227 & P4.1 & IVD12 & P17.1 & Leu 10 & $B 7 / 21$ \\
\hline \multicolumn{9}{|c|}{ \% inhibition of proliferative response } \\
\hline T4-31 & 76 & 98 & 85 & 99 & 4 & 0 & 0 & 0 \\
\hline GA1-5 & 100 & 100 & 11 & 100 & 8 & 0 & 0 & 1 \\
\hline BA1-7 & 100 & 99 & 63 & 99 & 0 & 6 & 7 & n.t. \\
\hline CB1-15 & 100 & 100 & 35 & 99 & 3 & 0 & 6 & 0 \\
\hline C2-1 & n.t. & 8 & n.t. & 7 & 70 & 16 & 4 & 2 \\
\hline $\mathrm{Cl}-19$ & 0 & 0 & 6 & 8 & 69 & 0 & 2 & 0 \\
\hline $\mathrm{C} 2-6$ & n.t. & 7 & 0 & 0 & 66 & 0 & 13 & 3 \\
\hline \multicolumn{3}{|c|}{ B. Clone } & \multicolumn{2}{|c|}{ Recognized specificity } & \multicolumn{2}{|c|}{ Encoding region } & \multicolumn{2}{|c|}{ Epitope } \\
\hline \multicolumn{3}{|c|}{ T4-31 } & \multicolumn{2}{|l|}{ Dw14 } & \multicolumn{2}{|c|}{ HLA-DR } & \multicolumn{2}{|c|}{ DR-T1 } \\
\hline \multicolumn{3}{|c|}{ GA1-5 } & \multicolumn{2}{|l|}{ Dw14 } & \multicolumn{2}{|c|}{ HLA-DR } & \multicolumn{2}{|c|}{ DR-T2 } \\
\hline \multicolumn{3}{|c|}{ BA1-7 } & \multicolumn{2}{|l|}{ Dw14 } & \multicolumn{2}{|c|}{ HLA-DR } & \multicolumn{2}{|c|}{ DR-T3 } \\
\hline \multicolumn{3}{|c|}{ CB1-15 } & \multicolumn{2}{|l|}{ Dw14/Dw10 } & \multicolumn{2}{|c|}{ HLA-DR } & \multicolumn{2}{|c|}{ DR-T4 } \\
\hline \multicolumn{3}{|c|}{ C2-1 } & \multicolumn{2}{|l|}{ Dw14 } & \multicolumn{2}{|c|}{ HLA-DQ } & \multicolumn{2}{|c|}{ DQ-T1 } \\
\hline \multicolumn{3}{|c|}{$\mathrm{C} 1-19$} & \multicolumn{2}{|l|}{ Dw14 } & \multicolumn{2}{|c|}{ HLA-DQ } & \multicolumn{2}{|c|}{ DQ-T2 } \\
\hline & \multicolumn{2}{|l|}{$\mathrm{C} 2-6$} & \multicolumn{2}{|l|}{ Dw14 } & \multicolumn{2}{|c|}{ HLA-DQ } & & \\
\hline
\end{tabular}

Distribution of $T$ cell recognition sites on $M H C$ class II molecules of patients with seropositive $R A$. Proliferative responses of these seven alloreactive $T$ cell clones were tested on the 23 patients described in Table I. The patients' cells were typed for the HLA-D specificity Dw 14 using PLT reagents. Of the 23 RA patients, only 2 of the 15 DR4-positive patients and none of the 8 DR4-negative patients induced a positive typing response for HLA-Dw14. Thus, the frequency of HLA-Dw14 defined by PLT was not increased in patients with seropositive RA. Although we would have predicted that only the two Dw14-positive patients' cells would stimulate this panel of $T$ cell clones, this was not the case. All cells tested from seropositive RA patients were seen by at least one of four of these clones. The $T$ cell epitopes defined by the panel of $T$ cell clones were expressed on different subpopulations of the patients. Although the specificities seen by the different clones were associated with HLA-Dw14 on the homozygous typing cell lines, the Dw14 associated T cell epitopes were present on cells from RA patients who were Dw14-negative and DR4-negative.

The determinants in DR-T2 and DR-T3 were found on only one patient (data not shown). In contrast, the $T$ cell epitopes DR-T1 and DR-T4 were seen frequently within the patients' group. The proliferative responses of clone T4-31 (recognizing DR-T1) and clone CB1-15 (recognizing DR-T4) on the panel of 23 RA patients are shown in Fig. $1 A$.

The determinant DR-T1 was identified in the majority of the DR4-positive patients, and, in addition, was expressed by two DR4-negative patients who shared the HLA-DR1 haplotype. A similar phenomenon was observed for the determinant DRT4. This epitope was expressed by most of the DR4-positive RA patients, but additionally by two DR4-negative RA patients both of whom typed Dw8. The DQ-encoded epitopes DQ-T1, DQ$\mathrm{T} 2$, and DQ-T3 were less frequent among the patients. DQ-T2 was found on a subset of DQ-T1 positive patients (data not presented). Proliferation of clone C2-1 and clone C2-6 in re- sponse to the $23 \mathrm{RA}$ patients is shown in Fig. $1 \mathrm{~B}$. The DQencoded determinants do not cluster with DR-T1 and DR-T4 epitopes, despite the fact that all of these determinants are concurrently expressed on the Dw14-positive homozygous typing lines (Table III). Patients who did not carry the HLA-DR4 DQw3 haplotype induced proliferation of the two DQ-specific $T$ cell clones $\mathrm{C} 2-1$ and $\mathrm{C} 2-6$, indicating that conventional serologic techniques do not identify $T$ cell recognition sites. Thus, $T$ cell epitopes, the combination of which defines the HLA-D specificity HLA-Dw14 (18), are very frequent but independently distributed in patients with seropositive RA. Each patient of the group of 23 RA patients tested expressed at least one of the following four allodeterminants: DR-T1, DR-T4, DQ-T1, and DQ-T3 (Fig. $1 A$ and $B$ ).

Differential blocking patterns of clone CB1-15 in DR4 and non-DR4 haplotypes. $\mathrm{CB} 1-15$ proliferated in response to two RA patients sharing the Dw8 haplotype. CB1-15 could be blocked by monoclonal antibodies specific for DR-encoded molecules on both DR4 and Dw8 haplotypes indicating that the epitope DR-T4 is located on a DR $\alpha-\beta$ dimer (Fig. 2). However, the blocking pattern on DR4-positive and DR4-negative (Dw8positive) stimulators was clearly different. Monoclonal antibody L227 most efficiently inhibited Dw8-positive targets, whereas it did not significantly effect the proliferative response of CB1-15 to Dw14-positive HTC. On Dw14-positive targets, P4.1 was the most effective antibody. The inhibition pattern on DR4-positive RA patients was indistinguishable from that on LS40 and Bin40. Thus, the epitope DR-T4 is present in a different configuration on the DR molecules of DR4-positive and DR4-negative RA patients' cells.

Distribution of the defined class II allodeterminants in nonrheumatoid individuals. The $\mathrm{T}$ cell epitopes defined by the seven clones were rare among non-DR4 nonrheumatoid individuals ( 2 out of 23) (data not presented). T4-31 proliferated in response to a donor typed DR1,5. C2-1 was reactive to stimulators of a 


$\begin{array}{ll}4,7 & 2,3 \\ 1,4 & 3 \\ 4,-- & 3 \\ 4,- & 2,3 \\ 4,5 & 3 \\ 4,5 & 3 \\ 4, w 8 & 3 \\ 4, w 9 & 3 \\ 1,4 & 1,3 \\ 1,4 & 1,3 \\ 1,4 & 1 \\ 4, w 10 & 1,3 \\ 3,4 & 2,3 \\ 4,- & 3 \\ 4, w 10 & 1,3 \\ 2, w 8 & 1,3 \\ 5, w 8 & 3 \\ 1,- & 1 \\ 1,7 & 1,2 \\ 2,7 & 1,2 \\ 2,7 & 1,2 \\ \text { w6,- } & 2,3 \\ 5, w 10 & 1,3\end{array}$
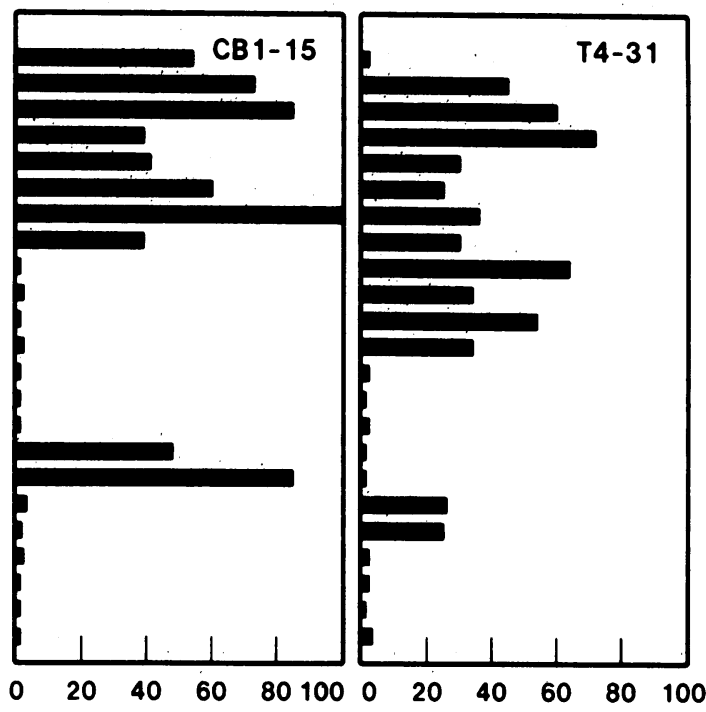

A

PERCENT RELATIVE RESPONSE

$\begin{array}{ll}\text { HLA-DR } & \text { HLA-DQ } \\ & \\ 4,7 & 2,3 \\ 1,4 & 3 \\ 4,- & 3 \\ 4,-- & 2,3 \\ 4,5 & 3 \\ 4,5 & 3 \\ 4, w 8 & 3 \\ 4, w 9 & 3 \\ 1,4 & 1,3 \\ 1,4 & 1,3 \\ 1,4 & 1 \\ 4, w 10 & 1,3 \\ 3,4 & 2,3 \\ 4,- & 3 \\ 4, w 10 & 1,3 \\ 2, w 8 & 1,3 \\ 5, w 8 & 3 \\ 1,- & 1 \\ 1,7 & 1,2 \\ 2,7 & 1,2 \\ 2,7 & 1,2 \\ \text { w6,- } & 2,3 \\ 5, w 10 & 1,3\end{array}$

$\begin{array}{lr} & \\ \text { DRw52 } & \text { DRw } \\ + & + \\ - & + \\ + & + \\ + & + \\ + & + \\ + & + \\ + & + \\ + & + \\ + & + \\ - & + \\ + & + \\ + & + \\ + & + \\ + & + \\ + & + \\ + & +\end{array}$
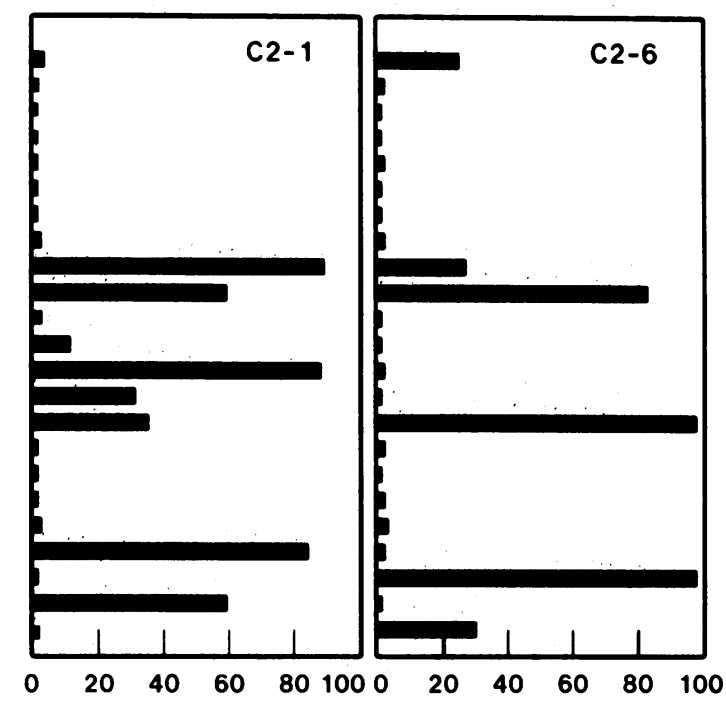

PERCENT RELATIVE RESPONSE

Figure 1. Proliferative responses of alloreactive T cell clones on cells from 23 patients with seropositive RA. Clone T4-31 and clone CB1-15 are reactive against $\mathrm{DR}$-encoded molecules $(A)$. Clone $\mathrm{C} 2-1$ and clone C2-6 are specific for DQ products $(B)$. Proliferative response was ob-

DR2, negative individual. 18 DR4-positive normal (nonrheumatoid) donors were tested for the presence of the determinants defined by the panel of alloreactive $T$ cell clones described above. As shown in Table VI, two clearly distinct groups could be differentiated: half of the individuals were negative for the four $T$ cell epitopes DR-T1, DR-T4, DQ-T1, and DQ-T3. The other $50 \%$ of the healthy donors expressed one or more of the $\mathrm{T}$ cell epitopes seen by the clones. Although determinants DR-T2 and DR-T3 were present on only one patient with seropositive RA, they had a similar frequency to DR-T1 and DR-T4 within the normal population (4 out of 18) and were coexpressed on targets positive for DR-T1 or DR-T4 (data not presented). tained as described in Methods. Bars represent the relative response induced by each individual stimulator when compared to the $\left[{ }^{3} \mathrm{H}\right]$ thymidine incorporation for Bin40 or LS40 (see Methods).

\section{Discussion}

The association of seropositive RA with HLA-DR4 is well established (10-13). This association has been interpreted as demonstration of a susceptibility factor which might be inherited within or in strong linkage to the HLA-D region. Genes within the HLA-D region encode cell surface molecules which are used by $T$ cells as restricting elements for the recognition of foreign antigens $(11,12)$. We addressed the question of whether patients with seropositive RA share MHC class II-encoded T cell restriction elements which might initiate a "pathogenic" immune response leading to a disease process manifested by a variety of 


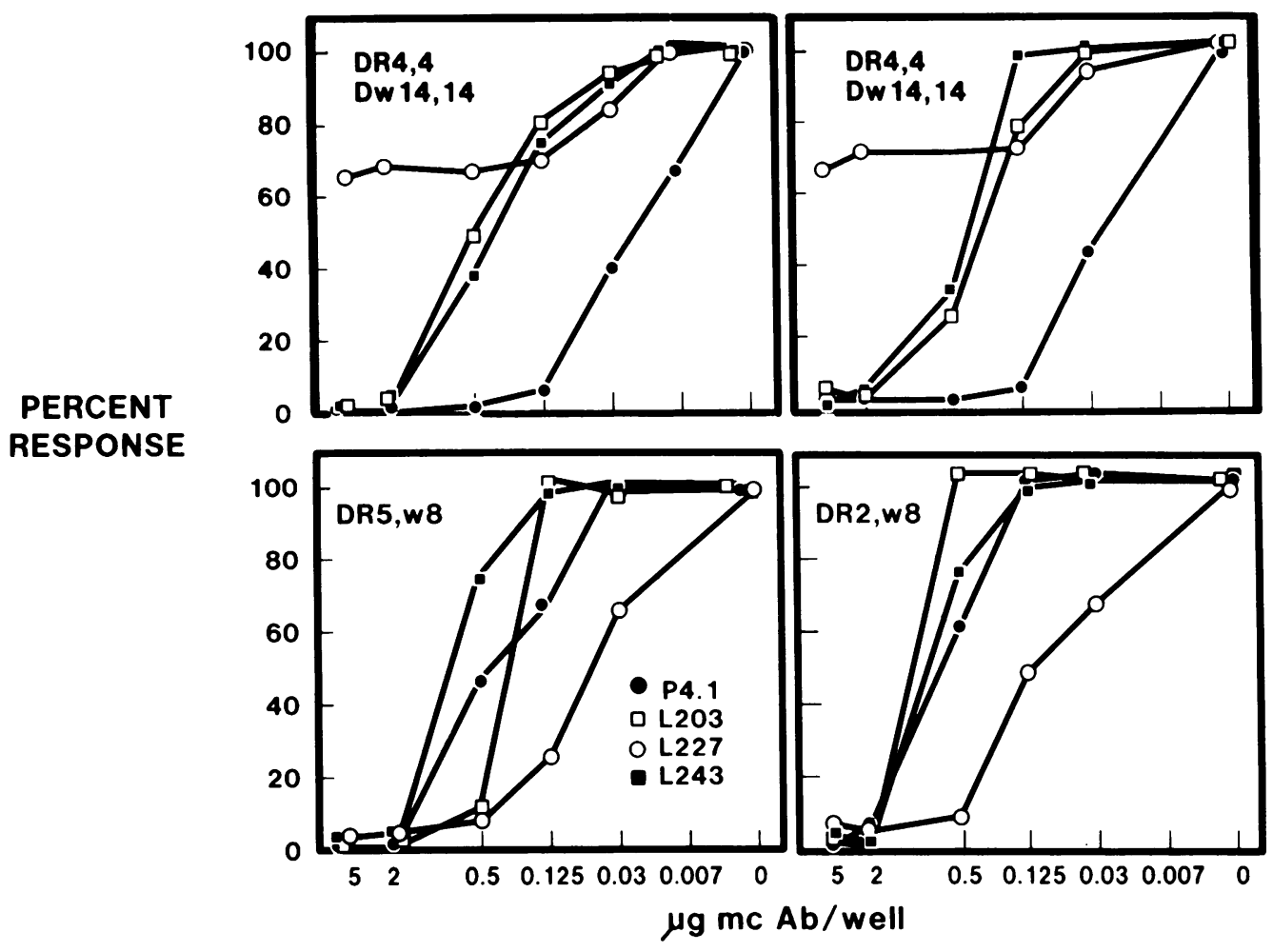

Figure 2. Distinct target inhibition pattern of Clone CB1-15 by monoclonal anti-DR antibodies on DR4-positive and DR4-negative targets. Alloantigen-induced proliferation was measured as described in Table III. Serial dilutions of monoclonal antibodies (5.0-0.007 $\mu \mathrm{g} /$ well) was added to target cells before the initiation of the cultures. Bin40 (DR4

autoimmune symptoms. Data presented in this manuscript suggest that there is a high frequency of HLA-Dw14-associated T cell epitopes present on cells from patients with seropositive RA irrespective of their serologic or cellular HLA type.

Previous techniques used to analyze class II antigens of patients with RA have included conventional serologic HLA-DR typing (11). The fact that the serotype HLA-DR4 has been differentiated by cellular typing into five subgroups suggested that the polymorphic structures of the HLA-D region seen by alloantisera were different from the determinants recognized by $T$ lymphocytes in MLR. The functional role of class II antigens in immune response involves their recognition by $T$ lymphocytes. Recent observations in our laboratory suggested that MHC class II allostimulating determinants are $\mathrm{T}$ cell restriction sites in antigen-specific responses (17). Thus, alloreactive $T$ lymphocytes should be appropriate reagents to analyze the immunologically relevant fine specificities of HLA-D molecules for HLA and disease associations. Interestingly, previous attempts to strengthen the HLA-DR4 association with RA by conventional cellular typing techniques have failed $(23,24)$. Although Dw4 was the predominant HLA-D specificity associated with RA, the association was less than the HLA-DR4 relationship. We have recently developed alloreactive human $\mathrm{T}$ cell clones to dissect HLA-D specificities. Using such clones, we were able to show that a positive typing response in mixed lymphocyte reactions giving rise to HLA-D assignment is elicited by the recognition of a combination of different $T$ cell epitopes (18). Thus, conventional typing is not sufficient to reflect the presence of single determinants. In order to look for shared HLA-D-encoded epitopes on cells from patients with seropositive RA, we generated a panel of alloreactive human $\mathrm{T}$ cell clones.

Dw14), LS40 (DR4 Dw14), and cells from two DR4-negative RA patients (DR2, w8 and DR5, w8) were used as stimulators. Results are presented as percent response calculated by dividing the antigen induced thymidine in the presence of antibodies by the uptake in the absence of antibody $\times 100$.

The T cell clones used in this study were established by stimulation of PBL against a B lymphoblastoid cell line from a patient with seropositive juvenile rheumatoid arthritis (JRA). This disease is similar to the adult form of seropositive RA. Class II molecules on the cells from the JRA patient had been previously characterized by detailed cellular typing and biochemical analysis (25). A panel of seven different $T$ cell clones was developed. Based on differences in target inhibition studies with monoclonal antibodies and distinct recognition patterns on a panel of stimulator cells, these clones defined different $T$ cell recognition sites related to HLA-Dw14 (Table VB). In a group of adult RA patients, the individual $T$ cell epitopes were very frequent; however, the concurrent expression of multiple determinants, equivalent to the cellular type HLA Dw14, was a rare event. Surprisingly, T cell epitopes DR-T1, DR-T4, DQ-T1, DQ-T2, and DQ-T3 were found on cells of patients that did not type HLA-DR4. About one-third of the patients were HLA-DR4-negative, but all of them were identified by at least one of four of the $T$ cell clones. The presence of an allodeterminant defined by the panel of clones used in this study in nonrheumatoid HLA-DR4-negative donors was rare ( 2 of 23 ).

The analysis of cells from a group of nonrheumatoid DR4positive individuals allowed their classification into two subgroups. Half of the donors did not express any of the determinants seen by this panel of clones, whereas the second half was characterized by a random distribution of the epitopes recognized by these clones. Thus, with regard to the presence of the described class II T cell recognition sites, rheumatoid patients clearly fall into a subgroup of DR4-positive haplotypes. Family studies have shown that affected and unaffected siblings share the DR4 haplotype (26-28). Such epidemiologic findings predict 
Table VI. Distribution Pattern of Four T Cell Epitopes on a Panel of Unrelated DR4 $4^{+}$Donors

\begin{tabular}{lllll}
\hline HLA-DR & DR-T1 & DR-T4 & DQ-T1 & DQ-T3 \\
\hline 3,4 & - & - & - & - \\
2,4 & - & - & - & - \\
4,4 & - & - & - & - \\
2,4 & - & - & - & - \\
$4, w 6$ & - & - & - & - \\
2,4 & - & - & - & - \\
4,7 & - & - & - & - \\
2,4 & - & - & - & - \\
4,4 & - & - & - & - \\
& - & - & + & - \\
$4, w 9$ & - & - & + & - \\
3,4 & + & + & - & - \\
4,7 & - & + & - & - \\
2,4 & + & + & - & - \\
4,4 & + & + & - & - \\
$4,-$ & - & - & + & + \\
$4, w 6$ & - & + & - & - \\
$4,-$ & - & + & + & + \\
$4, w 6$ & & & & \\
\hline
\end{tabular}

that genetic markers which are present in patients with RA must also be present in DR4-positive nonrheumatoid individuals substantiating the idea that rheumatoid arthritis is a multicausal disease including genetic and environmental factors (29).

For certain non-Caucasian ethnic groups, HLA-DR1 has been described as a genetic marker associated with seropositive RA $(30,31)$. Evidence that the phenotypes HLA-DR 1 and HLADR4 share determinants has been provided by the use of alloantiserum $\mathrm{Te} 23$ and different monoclonal antibodies $(32,33)$. Recently, a new MHC class II specificity, MC1, expressed on most DR1 and DR4 haplotypes has been reported to be associated with adult rheumatoid arthritis (34). The T cell recognition site DR-T1 was present in two non-DR4-positive patients sharing the HLA-DR1 haplotype. It is possible that shared T cell epitopes between DR 1 and DR4 explain increased frequencies of DR 1 in RA. The determinant DR-T4 was present in two patients who typed HLA-DRw8. No association between seropositive RA and HLA-DRw8 has yet been described.

In speculating about mechanisms that might give rise to $T$ cell epitopes appearing within different DR-frameworks, we favor the hypothesis that gene conversion-like events could be involved in shuffling small gene segments into the framework of different HLA-D molecules. This phenomenon was shown to have occurred in the mutant mouse bm12 (17). If, as in the mouse, such shuffled epitopes are functional in $T$ cell restriction (3537), it might explain the lack of stringent association between HLA molecules previously defined by serology or MLR with autoimmune diseases. Interestingly, we found that the $T$ cell epitope DR-T4 defined by clone CB1-15 is in a different configuration within the framework of a DR4 haplotype and the Dw8 haplotype, although DR-T4 is in both instances located on a DR molecule (Fig. 2). These studies suggested the possibility that the epitope DR-T4 may represent different placements of the epitope [hypervariable sites (38)] in the DR-molecule in DR4positive versus DR4-negative RA patients. Our model suggests that such shuffled epitopes might be involved in antigen recognition events leading to RA. Thus, by typing with $\mathrm{T}$ cell clones (not with antibodies or by MLR), we can define the "immunologically relevant" $T$ cell epitopes that may give rise to HLA and disease association. Such typing may eventually be used to define subgroups based upon these presumed genetic susceptibility markers which may allow therapeutic or prognostic assignment of patients among the heterogeneous population of patients with seropositive RA.

\section{Acknowledgment}

The authors would like to thank Dr. A. Zeevi, University of Pittsburgh for HLA-DR typing, Dr. R. Payne, Stanford University, and Dr. G. Nepom, Seattle, WA, for their gifts of HLA-DR-typed cell lines and PBL. We appreciate the expert secretarial assistance of Robyn Kizer and Diane O'Neill.

This study was supported by grants AI 18716 and AM 20610 from the National Institutes of Health. Dr. Goronzy is supported by the Deutsche Forschangsgemeinschaft, and Dr. Weyand is supported by the Arthritis Foundation.

\section{References}

1. Zinkernagel, R. M., and P. C. Doherty. 1974. Restriction of in vitro $\mathrm{T}$ cell mediated cytotoxicity in lymphocytic choriomeningitis within a syngeneic or semi-allogeneic system. Nature (Lond.). 248:701-720.

2. Thomas, D. W., U. Yamashita, and E. M. Shevach. 1977. The role of Ia antigens in T cell activation. Immunol. Rev. 35:97-120.

3. Thorsby, E., E. Berle, and H. Nouisainen. 1982. HLA-D region molecules restrict proliferative $\mathrm{T}$ cell responses to antigen. Immunol. Rev. 66:39-56.

4. Svejgaard, A., P. Platz, and L. P. Ryder. 1983. HLA and disease 1982-A survey. Immunol. Rev. 70:193-218.

5. Sasazuki, T., F. C. Grumet, and H. O. McDevitt. 1977. The association between genes in the major histocompatibility complex and disease susceptibility. Annu. Rev. Med. 28:425-452.

6. Engleman, E. G., C. J. Benike, F. C. Grumet, and R. L. Evans. 1981. Activation of human $T$ lymphocyte subsets: helper and suppressor cytotoxic $\mathrm{T}$ cells recognize and respond to distinct histocompatibility antigens. J. Immunol. 127:2124-2129.

7. Reinsmoen, N., and F. H. Bach. 1982. Five HLA-D clusters associated with HLA-DR4. Hum. Immunol. 4:249-258.

8. Dupont, B., D. W. Braun, Jr., E. J. Yunis, and C. B. Carpenter. 1980. HLA-D by cellular typing. In Histocompatibility Testing. P. I. Terasaki, editor. UCLA Tissue Typing Laboratory, Los Angeles. 229267.

9. Nepom, B. S., G. T. Nepom, E. Mickelson, P. Antonelli, and J. A. Hansen. 1983. Electrophoretic analysis of human HLA-DR antigens from HLA-DR4 homozygous cell lines: Correlation between $\beta$-chain diversity and HLA-D. Proc. Natl. Acad. Sci. USA. 80:6962-6966.

10. Stastny, P. 1978. Association of the B-cell allo antigen DRw4 with rheumatoid arthritis. N. Engl. J. Med. 298:869-871.

11. Stastny, P. 1980. Joint report on rheumatoid arthritis. In Histocompatibility Testing 1980. P. I. Terasaki, editor. UCLA Tissue Typing Laboratory, Los Angeles. 681-686.

12. McMichael, A. J., T. Sasazuki, H. O. McDevitt, and R. O. Payne. 1977. Increased frequency of HLA-CW3 and HLA-Dw4 in rheumatoid arthritis. Arthritis Rheum. 20:1037-1041.

13. Panayi, G. S., P. H. Wooley, and J. R. Batchelor. 1979. HLADRw4 and rheumatoid arthritis. Lancet. i:730.

14. Karr, R. W., G. E. Rodey, T. Lee, and B. D. Schwartz. 1980. Association of HLA-DRw4 with rheumatoid arthritis in black and white patients. Arthritis Rheum. 23:1241-1245.

15. Sasazuki, T., N. Ohta, Y. K. Nishimura, T. Abe, C. Abe, and Y. Shiokawa. 1981. Japanese rheumatoid arthritis and HLA. In New Horizons in Rheumatoid Arthritis. Y. Shiokawa, T. Abe, and Y. Yamauchi, editors. Excerpta Medica, Amsterdam. 247-254. 
16. Bias, W. B., S. H. Hsu, M. K. Pollard, J. Harvey, M. T. Lotze, F. C. Arnett, and M. B. Stevens. 1981. HLA-DR characterization of a Chippewa Indian subpopulation with high prevalence of rheumatoid arthritis. Hum. Immunol. 2:155-163.

17. Mengle-Gaw, L., S. Conner, H. O. McDevitt, and C. G. Fathman 1984. Gene conversion between murine class II major histocompatibility complex loci: functional and molecular evidence from the bm 12 mutant. J. Exp. Med. 160:1184-1194.

18. Weyand, C., J. Goronzy, and C. G. Fathman. 1986. Human T cell clones used to define functional epitopes on HLA Class II molecules. Proc. Natl. Acad. Sci. USA. In press.

19. Segall, M., N. L. Reinsmoen, J. Iwaki, and F. H. Bach. 1982. Determinants not correlated with HLA-Dw, DR, or SB detected by primed lymphocyte typing. Hum. Immunol. 4:239-248.

20. Morling, N., B. K. Jakobsen, P. Platz, L. P. Ryder, A. Svejgaard, and $\mathrm{M}$. Thomsen. 1981. Hum. Immunol. 2:333-340.

21. van Leeuwen, A., and J. J. van Rood. 1980. Description of B cell methods. In Histocompatibility Testing. P. I. Terasaki, editor. UCLA Tissue Typing Laboratory, Los Angeles. 278-279.

22. Terasaki, P. I. editor. 1980. Histocompatibility Testing. UCLA Tissue Typing Laboratory, Los Angeles.

23. Young, A., D. Jaraquemada, J. Awad, H. Festenstein, M. Corbett, F. C. Hay, and I. M. Roitt. 1984. Association of HLA-DR4/Dw4 and DR2/Dw2 with radiologic changes in a prospective study of patients with rheumatoid arthritis. Arthritis Rheum. 27:20-25.

24. Ollier, W., P. J. W. Venables, P. A. Mumford, R. N. Maini, J. Awad, J. Quemada, J. D'Amaro, and H. Festenstein. 1984. HLA antigen associations with extra-articular rheumatoid arthritis. Tissue Antigens. 24:279-291.

25. Nepom, B. S., G. T. Nepom, E. Mickelson, J. G. Schaller, P. Antonelli, and J. A. Hansen. 1984. Specific HLA-DR4-associated histocompatibility molecules characterize patients with seropositive juvenile rheumatoid arthritis. J. Clin. Invest. 74:287-291.

26. Nunez, G., S. Moore, G. V. Ball, E. R. Hurd, and P. Stastny. 1984. Study of HLA antigens in ten multiple-case rheumatoid arthritis families. J. Rheumatol. 11:129-135.
27. Bodmer, J., and W. Bodmer. 1984. Histocompatibility 1984. Immunol. Today. 5:251-254.

28. Khan, M. A., I. Kushner, and L. R. Weitkamp. 1983. Genetics of HLA-associated disease; rheumatoid arthritis. Tissue Antigens. 22: 182-185.

29. Miller, M. L., and D. N. Glass. 1981. The major histocompatibility complex antigens in rheumatoid arthritis and juvenile arthritis. Bull. Rheum. Dis. 31:21-23.

30. Schiff, B., Y. Mizrachi, S. Orgad, M. Yaron, and E. Gazit. 1982. Association of HLA-Aw31 and HLA-DR1 with adult rheumatoid arthritis. Ann. Rheum. Dis. 41:403-404.

31. Nichol, F. E., and J. C. Woodrow. 1981. HLA-DR antigens in Indian patients with rheumatoid arthritis. Lancet. i:220-221.

32. Bodmer, J. G., L. J. Kennedy, M. Aizawa, R. L. Dawkins, V. Lepage, M. C. Mazilli, P. Richiardi. 1984. HLA-D region monoclonal antibodies. In Histocompatibility Testing, 1984. E. D. Albert, M. P. Baur, and W. R. Mayr, editors. Springer-Verlag, Berlin. 217-236.

33. Colombani, J., and V. Lepage. 1984. HLA monoclonal antibody registry: third listing. Tissue Antigens. 24:209-214.

34. Duquesnoy, R. J., M. Marrari, S. Hackbarth, and A. Zeevi. 1984. Serological and cellular definition of a new HLA-DR associated determinant, MCl, and its association with rheumatoid arthritis. Hum. Immunol. 10:165-176.

35. Michaelides, M., M. Sandrin, G. Morgan, I. F. C. McKenzie, R. Ashman, and R. W. Melvold. 1981. Ir gene function in an I-A subregion mutant B6.C-H-2 ${ }^{\text {bm } 12}$. J. Exp. Med. 153:464-469.

36. Lin, C-C. S., A. S. Rosenthal, H. C. Passmore, and T. H. Hansen. 1981. Selective loss of an antigen-specific Ir gene function in the I-A mutant B6.C-H-2 ${ }^{\text {bm12 }}$ is an antigen presenting cell defect. Proc. Natl. Acad. Sci. USA. 78:6406-6410.

37. Hochman, P. S., and B. T. Huber. 1984. A class II gene conversion event defines an antigen-specific Ir gene epitope. J. Exp. Med. 160:19251930.

38. Bell, J., P. Estess, T. St. John, R. Saiki, D. L. Watling, H. A. Erlich, and H. O. McDevitt. 1985. DNA sequence and characterization of human class II major histocompatibility complex $\beta$ chains from the DR1 haplotype. Proc. Natl. Acad. Sci. USA. 82:3405-3409. 